\title{
A Note on the Inversion of Power Series
}

\section{Introduction.}

At one time or another most applied mathematicians are faced with the problem of calculating the coefficients of the series

$$
x=b_{1} y+b_{2} y^{2}+\cdots+b_{n} y^{n}+\cdots
$$

when given the coefficients of the series

$$
y=a_{1} x+a_{2} x^{2}+\cdots+a_{n} x^{n}+\cdots, \quad a_{1} \neq 0 .
$$

At such times there exists a choice between two long methods. The computer who is faced with this problem very of ten may derive explicit formulae for the desired coefficients and substitute directly. This method has the drawback usually encountered in substituting in formulae, namely that the computations are usually unsystematic and therefore become tedious and subject to many errors. Furthermore the formulae become extremely long and complicated for coefficients of appreciable order. ${ }^{1}$

The chief purpose of this paper lies in presenting a method which will be especially useful to the person who is unwilling to derive complicated formulae or undergo the ordeal of substituting in them. The method which will be presented will enable the computer to obtain $n$ coefficients of the inverse power series using only one page of computations with approximately $\frac{1}{2}(n+1)^{2}$ numbers. Besides being compact, this method has the advantage of being systematic. Furthermore similar methods can be easily obtained for most formal calculations with power series.

\section{Multiplication of Power Series.}

The fundamental part of the method of inversion is a simple device used to multiply power series. Because this method and its applications are not as widely known and appreciated as they should be, we shall indicate more properties of this method than is necessary for inversion.

If we are given

then

$$
\begin{aligned}
& y=a_{0}+a_{1} x+\cdots+a_{m} x^{m}+\cdots \\
& z=b_{0}+b_{1} x+\cdots+b_{m} x^{m}+\cdots
\end{aligned}
$$

where

$$
c_{m}=a_{0} b_{m}+a_{1} b_{m-1}+\cdots+a_{m} b_{0}=\sum_{i=0}^{m} a_{i} b_{m-i} .
$$

We write the coefficients $b_{i}$ in the first column and leave the second column for the $c_{i}$. We also take a strip of paper with the coefficients $a_{i}$ written from bottom to top. (See figure 1.)

We may calculate $c_{m}$ by adjusting the strip so that $a_{0}$ is adjacent to $b_{m}$ and then accumulating the products of all $a$ 's and $b^{\prime}$ 's which are adjacent. A special case of great importance is that where the $a_{i}$ and $b_{i}$ are coefficients of the same power series

$$
y=a_{0,1}+a_{1,1} x+\cdots+a_{m, 1} x^{m}+\cdots
$$

Then the $c_{i}$ are the coefficients of $y^{2}$. If we multiply the series with the 


$$
\mid \begin{gathered}
\dot{ } \\
\dot{a_{m}} \\
a_{m-1} \\
\dot{\cdot} \\
\dot{a_{1}} \\
a_{0} \\
\hline
\end{gathered}
$$

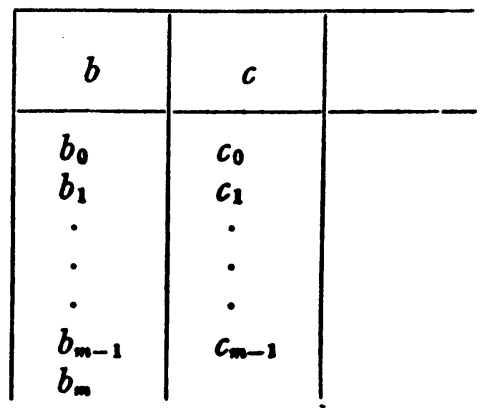

Figure 1.

coefficients $c_{i}$ by the original series we get the coefficients of $y^{2}$. Thus if we denote the coefficients of $y^{n}$ by means of

$$
y^{n}=a_{0, n}+a_{1, n} x+\cdots+a_{m, n} x^{m}+\cdots
$$

we obtain, by our method of multiplying power series, the coefficients $a_{m}$ n on a single sheet of paper. (See figure 2.)

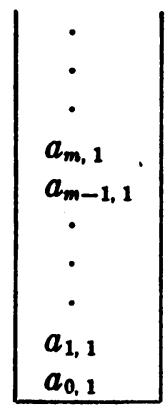

\begin{tabular}{|l|c|c|c|c|c}
\multicolumn{2}{c}{$y$} & \multicolumn{2}{c}{$y^{n}$} \\
\hline coef. of 1 & $a_{0,1}$ & $a_{0,2}$ & $\cdots$ & $a_{0, n}$ & $\cdots$ \\
coef. of $x$ & $a_{1,1}$ & $a_{1,2}$ & $\cdots$ & $a_{1, n}$ & $\cdots$ \\
$\cdot$ & $\cdot$ & $\cdot$ & & $\cdot$ & \\
$\cdot$ & $\cdot$ & $\cdot$ & & $\cdot$ & \\
coef. of $x^{m-1}$ & $a_{m-1,1}$ & $a_{m-1,2}$ & $\ldots$ & $a_{m-1, n}$ & $\ldots$ \\
coef. of $x^{m}$ & $a_{m, 1}$ & $a_{m, 2}$ & $\cdots$ & $a_{m, n}$ & $\cdots$ \\
$\cdot$ & $\cdot$ & $\cdot$ & & $\cdot$ & \\
$\cdot$ & $\cdot$ & $\cdot$ & & $\cdot$ & \\
\hline & $\cdot$ & $\cdot$ & & $\cdot$ &
\end{tabular}

Figure 2.

By use of the movable strip we may also calculate the quotient $z$ of the power series $u$ divided by that of $y$, assuming $a_{0} \neq 0 ;^{2}$ it is obvious that $b_{0}=c_{0} / a_{0}$. Furthermore if we are given $b_{0}, b_{1}, \cdots, b_{m-1}$ we can calculate $b_{m}$ by

$$
\sum_{i=1}^{m} a_{i} b_{m-i}=c_{m}, \quad b_{m}=\frac{1}{a_{0}}\left[c_{m}-\sum_{i=1}^{m} a_{i} b_{m-i}\right]
$$

where $\sum_{i=0}^{m} a_{i} b_{m-i}$ is obtained by means of the movable strip in an obvious manner.

Thus we can calculate the reciprocal $1 / y$ and then by successive multiplications $1 / y^{n}$; in fact we can calculate the power series $y^{n}$ for any real $n$ by making use of the binomial expansion,

$$
\begin{aligned}
y^{n}=\left[a_{0}+a_{1} x+\cdots+\right. & \left.a_{m} x^{m}+\cdots\right]^{n} \\
a_{0}^{n}[1+v]^{n} & =a_{0}^{n}\left[1+n v+\cdots+\left(\begin{array}{l}
n \\
r
\end{array}\right) v^{r}+\cdots\right] .
\end{aligned}
$$


Now we construct a table as in figure 2 for the function $v$. Above the top row, insert the numbers $\left(\begin{array}{l}n \\ r\end{array}\right)$ corresponding to the $r$-th column. Then the coefficient of $x^{m}$ in $y^{n}$ is $a_{0}^{n}\left[1+w_{m n}\right]$ where $w_{m n}=\sum\left(\begin{array}{l}n \\ r\end{array}\right) a_{m, r}$ is the accumulated product of the terms in the $m$-th row with the corresponding numbers $\left(\begin{array}{l}n \\ r\end{array}\right)$. Note that each sum is a finite sum because $v$ has no constant term and therefore $a_{m, r}=0$ for $r>m$.

The above process is a particular example of the evaluation of $u(x)$ $=f[g(x)]$ where $f$ and $g$ are power series. In general, if the coefficients for $g$ and $f$ are $a_{m, 1}$ and $b_{m}$ respectively, we have merely to replace $\left(\begin{array}{l}n \\ r\end{array}\right)$ in the above by $b_{r}$ and the coefficient of $x^{m}$ in $u$ is $b_{0}+w_{m}$ where $w_{m}=\sum_{r=1}^{\infty} b_{r} a_{m}$, .

The method of multiplying two power series in one variable by a strip of paper can be extended to the multiplication of power series in two or even more variables. Suppose where

$$
u=\sum a_{m, n} x^{m} y^{n}, \quad v=\sum b_{m, n} x^{m} y^{n}, \quad u v=w=\sum c_{m, n} x^{m} y^{n},
$$

$$
c_{m, n}=\sum a_{i, j} b_{m-i, n-j}
$$

To calculate $c_{m, n}$ we must consider instead of two columns $a_{i}, b_{i}$, the rectangular arrays $a_{i,}, b_{i, j}$. The extension is quite simple. We write the $a_{i, j}$ and $b_{i, j}$ on two cards as in figure 3 .

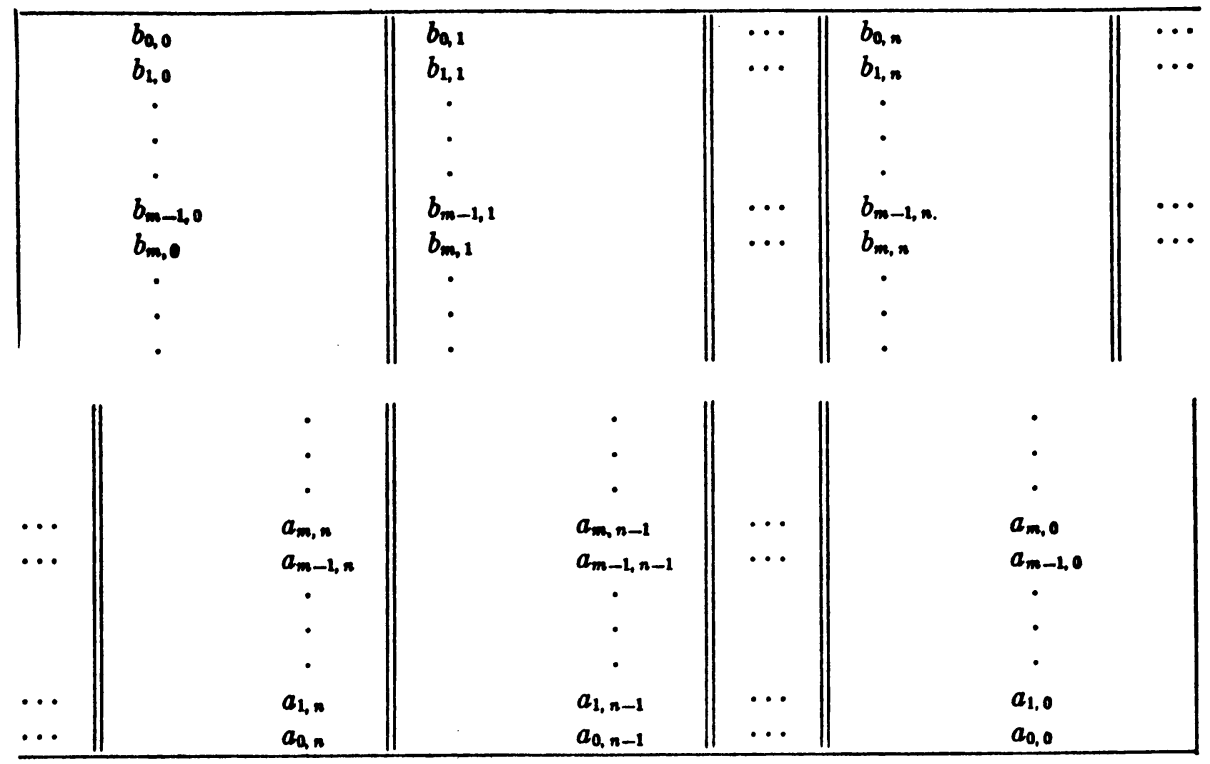

Figure 3.

The crosses indicate the portions of the $a$-card which are cut out, and it is quite evident that to get $c_{m, n}$ we have merely to place the $a$-card on the 
$b$-card so that $a_{0,0}$ corresponds to $b_{m, n}$ and to accumulate the products of corresponding terms.

It is noteworthy that in these formal operations with power series there is no need to restrict ourselves to series of positive powers only.

\section{Inversion.}

It is a simple step to compute the coefficients of the inverse series now. First we consider the case $y=a_{1,1} x+a_{2,1} x^{2}+\cdots$, i.e., $a_{0,1}=0$ and $a_{1,1} \neq 0$. Thus we can avoid dealing with all the terms above the diagonal for they will be zero. In fact our sheet would come out as in figure 4 after the addition of an extra column at the end and an extra row at the bottom of the sheet.

\begin{tabular}{c}
$\cdot$ \\
$\cdot$ \\
$a_{m, 1}$ \\
$a_{m-1,1}$ \\
$a_{m-2,1}$ \\
$\cdot$ \\
$\cdot$ \\
$\cdot$ \\
$a_{1,1}$ \\
0 \\
\hline
\end{tabular}

\begin{tabular}{|c|c|c|c|c|c|c|c|}
\multicolumn{4}{c}{$y$} & \multicolumn{1}{c}{$y^{2}$} & \multicolumn{1}{c}{$y^{m-1}$} & $y^{m}$ & \multicolumn{2}{c|}{$d_{i}$} \\
\hline coef. of 1 & 0 & 0 & $\cdots$ & 0 & 0 & $\cdots$ & \\
coef. of $x$ & $a_{1,1}$ & 0 & $\cdots$ & 0 & 0 & $\cdots$ & $d_{1}$ \\
coef. of $x^{2}$ & $a_{2,1}$ & $a_{2,2}$ & $\cdots$ & 0 & 0 & $\cdots$ & $d_{2}$ \\
$\cdot$ & $\cdot$ & $\cdot$ & & $\cdot$ & $\cdot$ & & \\
$\cdot$ & $\cdot$ & $\cdot$ & & $\cdot$ & $\cdot$ & & \\
coef. of $x^{m-1}$ & $a_{m-1,1}$ & $a_{m-1,2}$ & $\cdots$ & $a_{m-1, m-1}$ & 0 & $\cdots$ & $d_{m-1}$ \\
coef. of $x^{m}$ & $a_{m, 1}$ & $a_{m, 2}$ & $\cdots$ & $a_{m, m-1}$ & $a_{m, m}$ & $\cdots$ & \\
$\cdot$ & $\cdot$ & $\cdot$ & & $\cdot$ & $\cdot$ & & \\
$\cdot$ & $\cdot$ & $\cdot$ & & $\cdot$ & $\cdot$ & & \\
$\cdot$ & $\cdot$ & $\cdot$ & & $\cdot$ & $\cdot$ & & \\
\hline$b_{i}$ & $b_{1}$ & $b_{2}$ & & $b_{m-1}$ & $b_{m}$ & &
\end{tabular}

Figure 4.

Now, since $y=a_{1,1} x+a_{2,1} x^{2}+\cdots+a_{m, 1} x^{m}+\cdots$

$x=b_{1} y+b_{2} y^{2}+\cdots+b_{m} y^{m} \cdots$

$x=b_{1} a_{1,1} x$

$+b_{1} a_{2,1} x^{2}+b_{2} a_{2,2} x^{2}$

$+b_{1} a_{3,1} x^{3}+b_{2} a_{3,2} x^{8}+b_{8} a_{3,8} x^{8}$

$+b_{1} a_{m, 1} x^{m}+b_{2} a_{m, 2} x^{m}+b_{3} a_{m, 2} x^{m}+\cdots+b_{m-1} a_{m, m-1} x^{m}+b_{m} a_{m, m} x^{m} \cdots$

Equating coefficients we have

$$
\begin{aligned}
b_{1} a_{1,1} & =1, \quad b_{1}=1 / a_{1,1}, \quad \text { since } \quad a_{1,1} \neq 0, \\
b_{1} a_{2,1}+b_{2} a_{2,2} & =0, \quad b_{2}=-b_{1} a_{2,1} / a_{2,2}, \quad a_{m, m}=\left(a_{1,1}\right)^{m}
\end{aligned}
$$

Assuming we have $b_{1}, b_{2}, \cdots, b_{m-1}$, we use the $m$-th equation to get $b_{m}$

$$
b_{1} a_{m, 1}+b_{2} a_{m, 2}+\cdots+b_{m} a_{m, m}=0, \quad b_{m}=-d_{m} / a_{m, m} \text {, }
$$


where $d_{m}=b_{1} a_{m, 1}+b_{2} a_{m, 2}+\cdots+b_{m-1} a_{m, m-1}$ is obtained by an accumulation of the products of the $(m-1) b$ 's, which we know, by the corresponding $a$ 's in the row of coefficients of $x^{m}$.

Thus we can calculate as many of the coefficients of the inverse series as we wish by this method, being careful only to take a sheet of paper which is large enough, i.e., having $(r+1)$ rows and $(r+1)$ columns for $r$ coefficients. To recapitulate, this method permits us to calculate the coefficients of the inverse power series systematically and on one page. Furthermore in the calculations it requires only accumulations of products with the exception of $r$ divisions.

Now consider the inversion problem where the coefficient of $x$ is zero. If $z=d_{n} x^{n}+d_{n+1} x^{n+1}+\cdots$, we may, by the method described in 2 , obtain

$$
z^{1 / n}=a_{1,1} x+a_{2,1} x^{2}+\cdots,
$$

where $a_{1,1}=d_{n}^{1 / n} \neq 0$. Then we may obtain $x$ as a power series in $z^{1 / n}$.

H. Chernoff

Brown University

1 Franz Kamber, "Formules exprimant les valeurs des coefficients des séries de puissances inverses," Acta Math., v. 78, 1946, p. 193-204.

2 The case $a=0$ is easily handled by factoring out $x^{n}$ where $a_{n}$ is the first non-zero coefficient.

EdITORIal Note: A "movable strip" is extensively used by actuaries in their insurance and annuity calculations, in connection with their "commutation" columns. In actuarial literature there are frequent references to this "movable strip"; e.g., GEORGE KING, Institute of Actuaries' Text Book, part II, second ed., 1902, p. 392-393, 402.

\section{RECENT MATHEMATICAL TABLES}

For other RMT see ACM : Bibliography (Stibitz, NDRC, Zuse); OAC: Bibliography; N75 (Horton) and 79 (Katz); QR30.

425[A].-A. Adrian, Barème Forestier. Cubage des Bois abattus des Bois en grume d'après la Circonférence et le Diamètre et des Bois Équarris. Débit et Équarrissage des Bois. Cubage et Estimation des Bois sur Pied. Conversion du Volume réel. Paris, Editions Berger-Levrault, 54th thousand, 1944. iv, 214 p. $11.3 \times 17.5 \mathrm{~cm}$.

T. 1, p. 5-97 gives the volume in cubic meters, to $3 \mathrm{D}$, of round wood of circumference $c=25(1) 300$ centimeters and length $l=.25(.25) 16$ meters.

T. 2, p. 99-131, gives similar results for diameter $d=5(1) 100$ centimeters.

T. 3, p. 132-179, is for volumes of squared wood, $l=.25, .33, .5, .66, .75,1(1) 20$ meters, and cross sections $5 \times 5(1) 11$ up to $50 \times 50(1) 55,100$ centimeters.

T. 4, p. 180-185, by three different methods of "squaring" round wood, from $c=32$, $d=10,(7 \times 7,8 \times 8,8 \times 9)$ to $c=300, d=95 \frac{1}{2}(67 \times 68,75 \times 75,90 \times 91)$.

Miscellaneous small tables p. 190-212.

426[A].-R. C. Morris, "Table of multiples of the square root of three," Electrical World, v. 125, June 8, 1946, p. 108-109. $21.6 \times 28.8 \mathrm{~cm}$.

This is a table of $N \sqrt{3}$, where $\sqrt{3}$ is taken as $1.73205 ; N=[1(.01) 9.99 ; 4 \mathrm{D}]$. In MARCEL Bols, Tables Numeriques Universelles, Paris, 1947, p. 184-185, are 6D tables of $N x^{t} / D$ for $x=2,3,5, N$ or $D=1(1) 10$. 